\title{
Ingestion of thioproline suppresses rat esophageal adenocarcino- genesis caused by duodenogastroesophageal reflux
}

\author{
SHOZO SASAKI ${ }^{1}$, KOICHI MIWA ${ }^{2}$, TAKASHI FUJIMURA $^{1}$, MASARU OBA $^{1}$, \\ TOMOHARU MIYASHITA ${ }^{1}$ and SHINICHI KINAMI ${ }^{1}$ \\ ${ }^{1}$ Gastroenterologic Surgery, Department of Oncology, Division of Cancer Medicine, Graduate \\ School of Medical Science, Kanazawa University, 13-1 Takaramachi, Kanazawa 920-8641; \\ ${ }^{2}$ Department of Surgery, Toyama Rosai Hospital, 992 Rokuro-maru, Uozu 937-0042, Toyama, Japan
}

Received March 15, 2007; Accepted July 6, 2007

\begin{abstract}
Duodenogastroesophageal reflux causes esophageal adenocarcinoma in rats without the use of a carcinogen. This etiology is unclear, but may be associated with endogenous nitrosation in the gastrointestinal tract. Thioproline (TPRO) is an effective nitrite-trapping agent and blocks endogenous nitrosation. We investigated how ingested TPRO affected esophageal adenocarcinogenesis in rats with duodenogastroesophageal reflux (DGER) or gastroesophageal reflux (GER). A series of 200 male Fischer 344 rats received surgery to induce reflux of duodenogastric contents or gastric contents alone into the esophagus. The rats were separated into two divisions according to the surgical procedure employed (DGER or GER), and each division was further subdivided into two groups: one group was fed a special diet (CRF-1 containing $0.5 \%$ of TPRO); the other group was fed a standard diet (CRF-1). The rats were given no carcinogen and sacrificed at ten-week intervals from the 25 th to the 45 th week after surgery. Pathological examination was carried out using hematoxylin-eosin or immunohistochemical staining. Erosion, regenerative thickening, basal cell hyperplasia and columnarlined epithelium (CLE) were found in both groups of the DGER rats. Adenocarcinoma (AC) appeared only in the DGER rats sacrificed at 35 and 45 weeks following surgery. The incidence of $\mathrm{AC}$ at the 45th week was significantly lower in
\end{abstract}

Correspondence to: Dr Koichi Miwa, Toyama Rosai Hospital, 992 Rokuro-maru, Uozu, Toyama 937-0042, Japan

E-mail: kmiwa@toyamah.rofuku.go.jp

Abbreviations: NOC, $N$-nitroso compound; TPRO, thioproline, (thiazolidine-4-carboxylic acid); iNOS, inducible nitric oxide synthase; DGER, duodenogastroesophageal reflux; GER, gastroesophageal reflux; CLE, columnar-lined epithelium; AC, adenocarcinoma

Key words: thioproline, duodenogastroesophageal reflux, columnarlined epithelium, esophageal adenocarcinogenesis, $N$-nitroso compound the group of rats fed the diet containing TPRO, as compared to those fed the standard diet, whereas the incidences of CLE were the same for both groups. iNOS protein and nitrotyrosine protein were identified in the CLE and macrophages of the DGER group using immunohistochemical staining. There were no remarkable pathological changes in the esophagi of the rats which underwent the GER procedure. In conclustion, TPRO has an inhibitory effect on esophageal reflux-induced adenocarcinogenesis in rats in that it prevents the progression from CLE to $\mathrm{AC}$.

\section{Introduction}

The incidence of esophageal adenocarcinoma (AC) has been increasing not only in Western countries $(1,2)$ but also in East Asia (3). Esophageal AC occurs in the lower esophagus developing from the columnar-lined epithelium (CLE) known as Barrett's esophagus (4). Barrett's esophagus and esophageal AC are closely associated with duodenogastroesophageal (DGER) reflux (5-8). Rat experiments demonstrated that duodenal contents cause esophageal carcinoma without exposure to carcinogens (9-13), whereas gastric contents do not (9). Though the etiology of esophageal $\mathrm{AC}$ is unclear, Mirvish et al suggested that the human esophageal AC and Barrett's esophagus was initiated by nitrosoamine (14).

Endogenous $N$-nitroso compounds (NOCs) are produced by chemical reactions between amine or amide precursors and nitrites generated by nitrates $(15,16)$. NOCs are produced by acid-mediated nitrosation at low $\mathrm{pH}$ levels in the stomach (17). They can also be produced at neutral $\mathrm{pH}$ levels by intestinal bacteria possessing nitrate reductase and nitrosating enzymes via nitric oxide formation (18-20). It has been shown that free radicals mediate reflux esophagitis and Barrett's esophagus (21-23). Increased expression of inducible nitric oxide synthase (iNOS) is observed in not only esophageal squamous cell carcinoma but also Barrett's esophagus and subsequent AC $(24,25)$. Thus, nitric oxide might play an important role in esophageal carcinogenesis.

Thiazolidine-4-carboxylic acid (thioproline, TPRO) is a cyclic sulfur-containing amino acid that is a condensation product of cysteine and formaldehyde (26). TPRO rapidly 
traps nitrites in the human body (27) and changes into $\mathrm{N}$-nitrosothiazolidine-4-carboxylic acid (NTPRO) (Fig. 1). NTPRO is not carcinogenic and is excreted in the urine without further metabolism. Thus TPRO acts as a nitrite scavenger, rendering carcinogenic $N$-nitroso compounds inactive. Additionally, TPRO may act as an intracellular sulfhydryl antioxidant and as a scavenger of free radicals (28). Tahira et al have shown that TPRO suppresses carcinogenesis induced by $N$-benzylmethylamine and nitrites (29). Our previous investigation showed that the gastric carcinogenesis induced by duodenogastric reflux in rats was inhibited by ingested TPRO (30). Kumagai et al, our colleagues, reported the suppressing effect of TPRO on esophageal adenocarcinogenesis in rats (31).

Progression of esophageal adenocarcinogenesis is divided into 3 parts: esophagitis, CLE, and AC (32). The aim of this study is to investigate which component of the esophageal metaplasia-adenocarcinoma sequence in rats is suppressed by TPRO.

\section{Materials and methods}

Animals and experimental environment. Two hundred male Fischer 344 rats weighing $\sim 180 \mathrm{~g}$ each were housed three to a cage and maintained under conditions of $22 \pm 3 \%$ room temperature and $55 \pm 5 \%$ humidity with a 12 -h light-dark cycle.

Chemicals and diets. TPRO was purchased from ICN Biochemicals Inc. (OH, USA). CRF-1 (Charles River Co., Tokyo, Japan) was used as the standard diet. TPRO was added at the concentration of $0.5 \%(\mathrm{~W} / \mathrm{W})$ to the CRF-1.

Surgical procedure. After fasting for $24 \mathrm{~h}$, the rats received upper abdominal incisions under diethyl-ether inhalation anesthesia (Fig. 2).

Duodenogastroesophageal reflux (DGER). After both vagus nerves were preserved, the abdominal esophagus was transected under the diaphragm, and the distal cut end was closed with sutures. The esophageal stump was then anastomosed end-to-side to a loop of jejunum $4 \mathrm{~cm}$ distal to the ligament of Treitz in an ante-colic manner. This procedure allowed duodenogastric contents to flow back into the esophagus.

Gastroesophageal reflux (GER). The duodenum was cut off near the pylorus, and the distal duodenal stump was closed. The jejunum was transected $\sim 4 \mathrm{~cm}$ distal to the ligament of Treitz, and the distal, cut end was closed with sutures. The proximal duodenal stump was anastomosed end-to-side with the distal jejunum near the sutures. After both vagus nerves were preserved, the abdominal esophagus was transected below the diaphragm, and the distal cut end was closed with sutures. The esophageal stump was anastomosed end-to-side to the distal jejunum $\sim 1 \mathrm{~cm}$ distal to the gastro-jejunostoma. Finally, the proximal jejunal cut end was anastomosed endto-side with the jejunal loop $\sim 4 \mathrm{~cm}$ distal to the esophagojejunostoma. This surgery permitted only gastric contents to flow back into the esophagus.

Every intestinal anastomosis was carried out with 4 interrupted stitches through all the intestinal layers in a single

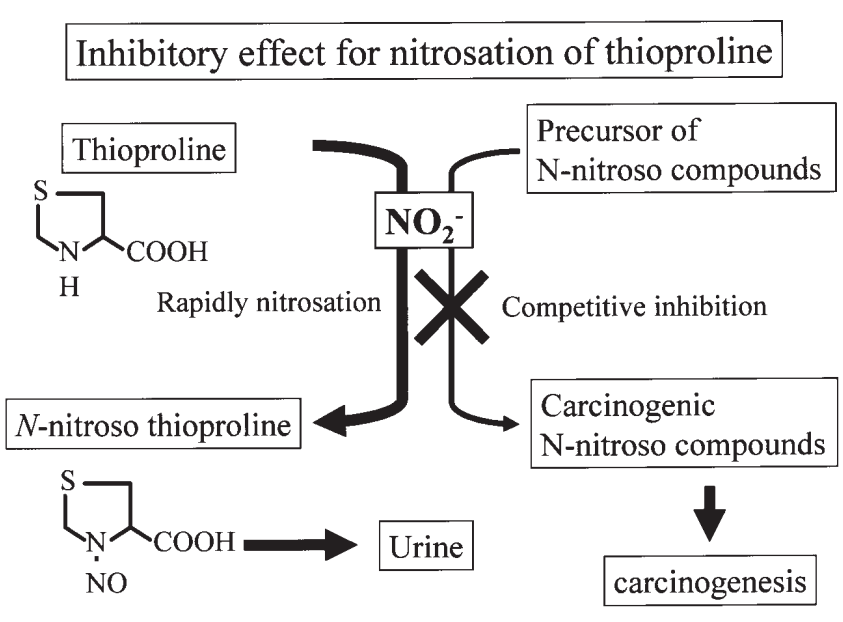

Figure 1. Inhibitory effect of thioproline (TPRO). TPRO is rapidly nitrosated and prevents the formation of NOC by trapping nitrites in the human body. TPRO is nitrosated to nitrosothioproline, which is non-mutagenic, and excreted in the urine.

line using 7-0 polypropylen-monofilament sutures. After the operation, the animals were allowed to drink immediately but continued fasting for 1 day. The rats were weighed every 4 weeks throughout the experiment. They were given no carcinogen, and sub-groups were sacrificed every 10 weeks from the 25 th week until the 45 th week after surgery.

Experimental groups. The animals were divided into 2 divisions according to the surgical procedure employed - the DGER division and the GER division - and each division was subdivided into 2 groups according to the diets administered as follows:

DGER division. Group A: animals fed a special diet (CRF-1 containing $0.5 \%$ TPRO) following the DGER procedure (40 animals); Group B: animals fed a standard diet (CRF-1) following the DGER procedure (60 animals).

GER division. Group C: animals fed a special diet (CRF-1 containing $0.5 \%$ of TPRO) following the GER procedure (40 animals); Group D: animals fed a standard diet (CRF-1) following the GER procedure (60 animals).

Pathological evaluation. The rats were sacrificed using diethylether inhalation and their abdomens were opened. The afferent and efferent jejunal loops were cut off and the esophagus was transected at the level of the thyroid cartilage. Then the esophagus and anastomosed jejunum were removed.

The esophagus was opened longitudinally and spread on a cork plate with the mucosal side up. After having been fixed with $10 \%$ formalin solution for $24 \mathrm{~h}$, the esophagus was cut at 3-mm intervals along the longitudinal section and embedded in paraffin. Sections of each block $(4 \mu \mathrm{m})$ were prepared for pathological assessment with hematoxylin-eosin and immunohistochemical staining.

Definition of the pathological findings. The esophageal histological findings were classified in accordance with the descriptions by Miwa et al (10).

Erosion. Defect of the epithelium with inflammatory cell infiltration. 
DGER

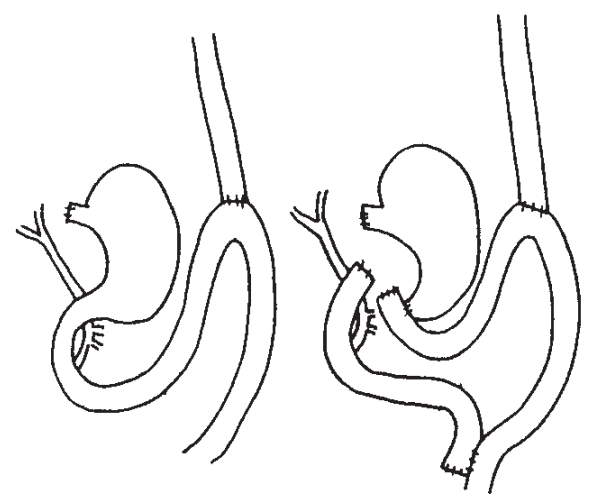

Figure 2. A surgical model for esophageal adenocarcinoma through reflux. In the duodenogastroesophageal reflux (DGER) case, end-to-side esophagojejunostomy to the jejunum $4 \mathrm{~cm}$ distal to the ligament of Treitz was performed to induce the return flow of gastroduodenal contents back into the esophagus. In the gastroesophageal reflux (GER) case, the end-to-side esophagojejunostomy to the jejunum $1 \mathrm{~cm}$ distal to the gastrojejunostoma and end-to-side anastomosis to the jejunal loop $4 \mathrm{~cm}$ distal to the esophagojejunostoma was made to induce the flow of gastric contents back into the esophagus.

Regenerative thickening. This condition is marked by esophageal epithelial thickening more than double the thickness of the normal epithelium with acanthosis, abnormal extension of papilla towards the mucosal surface and parakeratosis. The stratified structure of the epithelium is not disturbed.

Basal-cell hyperplasia. In this condition the basal layer in the squamous epithelium occupies $>15 \%$ of the full thickness of the squamous epithelial layer. It may contain intramural cysts. The stratified structure of the epithelium is preserved.
$C L E$. The esophageal squamous epithelium is replaced by columnar-lined epithelium with brush borders and goblet cells.

$A C$. Carcinoma is defined as an epithelial growth with cellular and structural atypism, invading into the submucosal layer. AC is a dysplastic glandular cell growth with both atypism and invasiveness, and is composed of tubular or papillary carcinoma or mucinous carcinoma.

Immunohistochemical analysis for iNOS and nitrotyrosine. Immunohistochemical staining for iNOS and nitrotyrosine in the DGER division were performed on the esophageal sections of the rats sacrificed at 45 weeks after surgery, using a Dako EnVision ${ }^{\mathrm{TM}}$ system (Dako, Tokyo, Japan). The sections were deparaffinized and incubated with $2 \mathrm{~N} \mathrm{HCl}$ for $30 \mathrm{~min}$ and neutralized with $1 \mathrm{~N}$ sodium borate for DNA denaturation. Then all the sections were heated with microwaves in a $0.01 \mathrm{~mol} / 1$ citrate buffer for $10 \mathrm{~min}$. The endogenous peroxidase activity in the tissue was quenched in methanol containing $0.3 \%$ hydrogen peroxide for $20 \mathrm{~min}$, and then each section was placed into protein block serum-free (Dako) for blocking the sections. The sections were incubated with mouse monoclonal antibodies to iNOS (Santa Cruz, CA, USA) and nitrotyrosine (EMD Bioscience Inc., USA) overnight at $4^{\circ} \mathrm{C}$. Dako EnVision labeled polymer (Dako), which is the secondary antibody to mouse and rabbit immunoglobulin combined with dextran marked with peroxidase, was used to detect the immunoreactivity. The antibody complexes were visualized by incubation with 3,3'-diaminobenzidine tetrahydrochloride. Sections were counterstained with hematoxylin staining.

Statistical analysis. The Fisher's exact test was used for statistical analysis of the incidence of pathological findings. $\mathrm{P}$-values of $<0.01$ were considered significant.

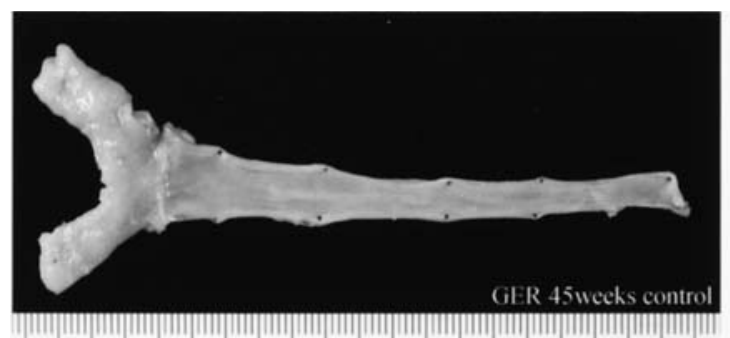

c

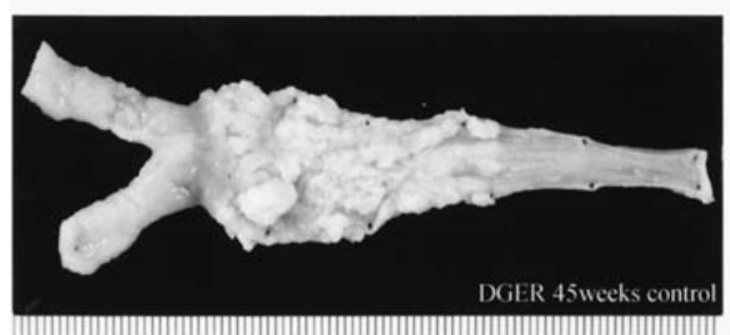

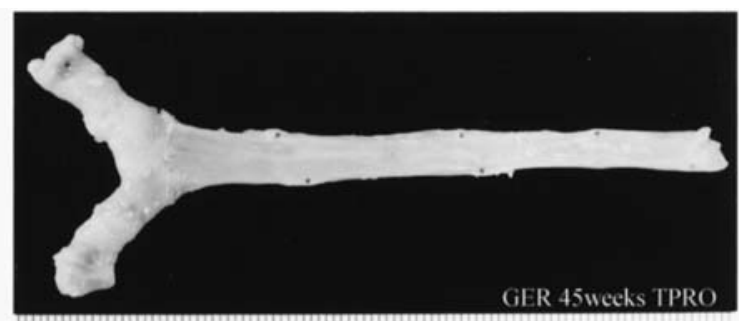

b

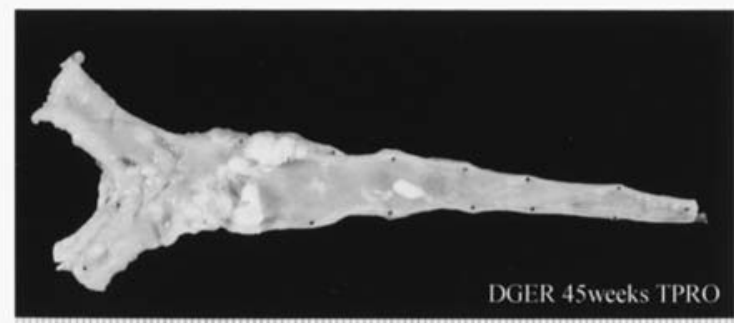

Figure 3. Macroscopic findings. There was very little erosion and only slight wall thickening of the esophagi of rats in both groups of the GER division at 45 weeks after operation ( $\mathrm{a}$ and b). In both groups A and B, the esophageal walls of all animals revealed uneven surfaces with thickening of the wall and upper and middle esophageal dilatation (c and d). Most of the rats exhibited stenosis and elevated lesions in the lower or middle esophagus due to ulceration or carcinoma in both groups of the DGER division at all observation weeks after operation (c and d). These findings were more obvious in group A than in group B (c and d), and these findings increased in later weeks for both groups. 
Table I. The incidence of pathological findings for rats with duodenogastroesophageal reflux.

\begin{tabular}{|c|c|c|c|c|c|c|}
\hline \multirow[b]{3}{*}{$\begin{array}{l}\text { Pathological } \\
\text { findings }\end{array}$} & \multicolumn{6}{|c|}{ DGER } \\
\hline & \multicolumn{2}{|c|}{25 weeks } & \multicolumn{2}{|c|}{35 weeks } & \multicolumn{2}{|c|}{45 weeks } \\
\hline & $\begin{array}{l}\text { TPRO } \\
(\mathrm{n}=5)\end{array}$ & $\begin{array}{c}\text { Control } \\
(\mathrm{n}=10)\end{array}$ & $\begin{array}{l}\text { TPRO } \\
(\mathrm{n}=6)\end{array}$ & $\begin{array}{l}\text { Control } \\
(\mathrm{n}=9)\end{array}$ & $\begin{array}{l}\text { TPRO } \\
(\mathrm{n}=20)\end{array}$ & $\begin{array}{l}\text { Control } \\
(\mathrm{n}=27)\end{array}$ \\
\hline ERO & $\begin{array}{c}5 \\
(100 \%)\end{array}$ & $\begin{array}{c}10 \\
(100 \%)\end{array}$ & $\begin{array}{c}6 \\
(100 \%)\end{array}$ & $\begin{array}{c}9 \\
(100 \%)\end{array}$ & $\begin{array}{c}20 \\
(100 \%)\end{array}$ & $\begin{array}{c}27 \\
(100 \%)\end{array}$ \\
\hline RT & $\begin{array}{c}5 \\
(100 \%)\end{array}$ & $\begin{array}{c}10 \\
(100 \%)\end{array}$ & $\begin{array}{c}6 \\
(100 \%)\end{array}$ & $\begin{array}{c}9 \\
(100 \%)\end{array}$ & $\begin{array}{c}20 \\
(100 \%)\end{array}$ & $\begin{array}{c}27 \\
(100 \%)\end{array}$ \\
\hline $\begin{array}{l}\text { Basal cell } \\
\text { hyperplasia }\end{array}$ & $\begin{array}{c}5 \\
(100 \%)\end{array}$ & $\begin{array}{c}10 \\
(100 \%)\end{array}$ & $\begin{array}{c}6 \\
(100 \%)\end{array}$ & $\begin{array}{c}9 \\
(100 \%)\end{array}$ & $\begin{array}{c}20 \\
(100 \%)\end{array}$ & $\begin{array}{c}27 \\
(100 \%)\end{array}$ \\
\hline CLE & $\begin{array}{c}2 \\
(40 \%)\end{array}$ & $\begin{array}{c}5 \\
(50 \%)\end{array}$ & $\begin{array}{c}5 \\
(83 \%)\end{array}$ & $\begin{array}{c}8 \\
(89 \%)\end{array}$ & $\begin{array}{c}17 \\
(85 \%)\end{array}$ & $\begin{array}{c}24 \\
(89 \%)\end{array}$ \\
\hline $\mathrm{AC}$ & $\begin{array}{c}0 \\
(0 \%)\end{array}$ & $\begin{array}{c}0 \\
(0 \%)\end{array}$ & $\begin{array}{c}0 \\
(0 \%)\end{array}$ & $\begin{array}{c}1 \\
(11 \%)\end{array}$ & $\begin{array}{c}1^{\mathrm{a}} \\
(5 \%)\end{array}$ & $\begin{array}{c}12 \\
(44 \%)\end{array}$ \\
\hline
\end{tabular}

${ }^{a} \mathrm{P}<0.01$ for adenocarcinoma in rats fed thioproline vs. the control group rats sacrificed at 45 weeks.

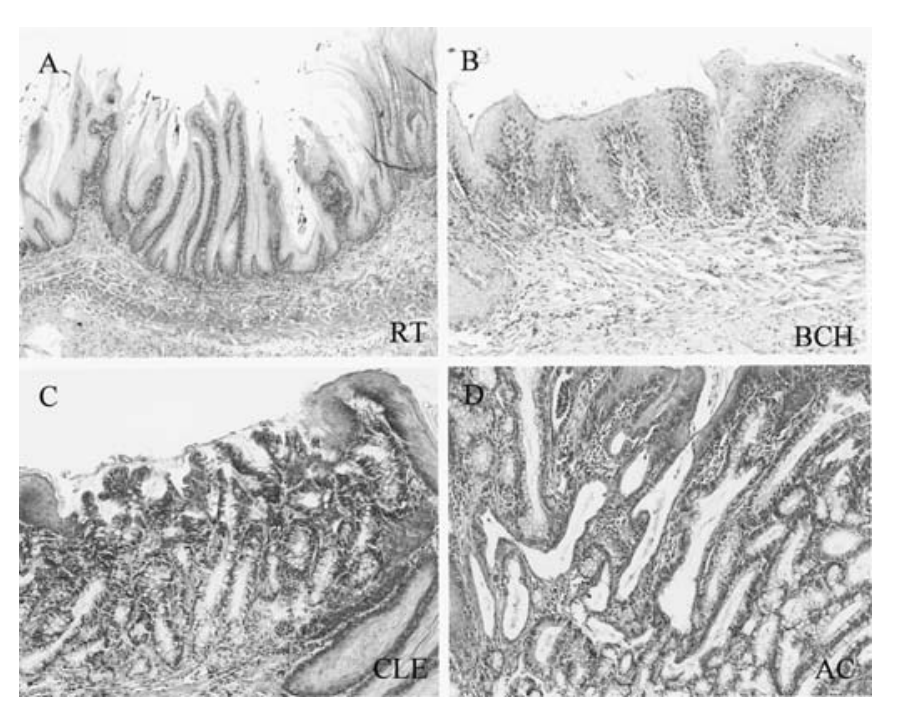

Figure 4. Histological findings. Regenerative thickening (A) and basal cell hyperplasia (B) were present in all groups. Columnar-lined epithelium (C) and adenocarcinoma (D) were found in the lower third of the esophagi in both groups of the DGER division.

\section{Results}

General observations. The number of examined rats sacrificed at the 25th, 35th and 45th weeks after surgery was: 5, 6 and 20 from group A; 10, 9 and 27 from group B; 5, 6 and 17 from group $C$; and 7,10 and 30 from group $D$, respectively. Twenty-three rats in the DGER division died of malnutrition and esophageal stenosis complicated by reflux esophagitis. Twenty-five rats in the GER division died within a week after surgery.

There were no significant differences in pre-operative average body weight $(\mathrm{g})$ (mean $\pm \mathrm{SD}$ ) among the groups. The average body weights (g) (mean \pm SD) at the 45 th week after surgery exhibited no significant differences between the groups A $(229 \pm 58)$ and B $(229 \pm 42)$ or between the groups C $(320 \pm 35)$ and D $(319 \pm 29)$, respectively. However, the body weights $(\mathrm{g})$ in the groups A and B were significantly lower than the groups $\mathrm{C}$ and $\mathrm{D}$, respectively.

Macroscopic findings. There were no remarkable macroscopic changes in the esophagi of the rats in the GER division at any examined week. On the other hand, in the DGER division, the lower portion of the esophagi revealed uneven surfaces and wall thickening whereas the middle and upper portions were dilated in all rats at observed week.

Mucosal lesions such as ulceration and protruded lesions in the lower esophagi in groups A and B were found with incidences of 50 and $40 \%$ in the 25 th week, 89 and $83 \%$ in the 35 th week, and 92 and $80 \%$ in the 45 th week, respectively. The incidence of the lesions increased in the later weeks but was almost the same for both groups in each week observed (Fig. 3).

Histological changes. There were slight histological changes including erosion and regenerative thickening in the GER division. Histological changes in the DGER division are shown in Table I. Erosion, regenerative thickening and basal cell hyperplasia were present in all rats at each examined week (Fig. 4). CLE was observed in both groups A and B, with incidences of 40 and $50 \%$ in the 25th week, 83 and $89 \%$ in the 35 th week, and 85 and $89 \%$ in the 45 th week after surgery, respectively (Fig. 4). Observations during the same weeks revealed the incidence of CLE was almost the same between both groups. AC appeared in the lower esophagus in groups A and $\mathrm{B}$, and the incidence was 0 and $0 \%$ in the 25th week, 0 and $11 \%$ in the 35 th week, and 5 and $44 \%$ in the 45 th week, respectively (Fig. 4). The occurrence of AC in group A was 

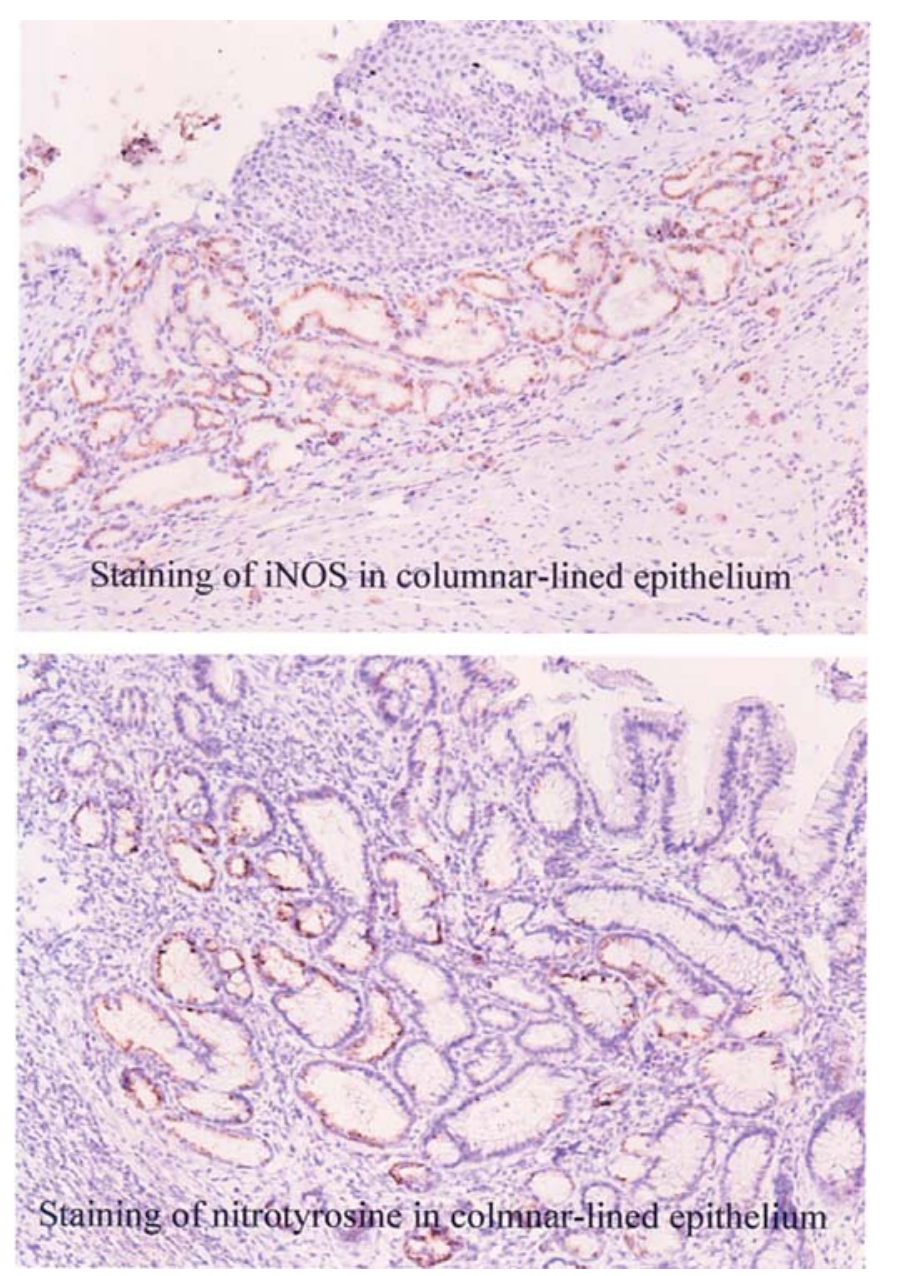

Figure 5. Imunohistochemical staining. Immunohistochemical staining for iNOS and nitrotyrosine were performed on esophageal sections from rats in the DGER division sacrificed at 45 weeks after surgery. Staining of iNOS and nitrotyrosine were seen in the CLE and stromal macrophages.

significantly lower than in group B at 45 weeks after surgery $(\mathrm{p}<0.01)$.

Immunohistochemical staining. Though iNOS and nitrotyrosine proteins were not detected in AC, they were overexpressed in CLE and its stromal macrophages (Fig. 5). No difference was found in the staining between groups $\mathrm{A}$ and $\mathrm{B}$.

\section{Discussion}

The present investigation reconfirmed that esophageal AC developed in rats with DGER, but not in those with GER. In addition, it was shown that duodeno-esophageal reflux alone can develop AC in rat esophagi (32). These findings suggest that regurgitated duodenal contents probably contain a carcinogenic substance which induces this carcinogenesis. A few reports suggest that duodenal juice might be carcinogenic. Mirvish (33) hypothesized that the intragastric formation of nitrosamides by the acid-catalyzed reaction of amides with nitrites is a plausible etiological factor in the development of human gastric cancer. Calmels et al described that endogenous nitrosation may be caused by bacteria that can carry out denitrification in rat stomach with achlorhydria (18). Busby et al indicated that two nitrosated bile acid conjugates, $N$-nitrosoglycocholic acid and $N$-nitrosotaurocholic acid, were hepatocarcinogenic in rats (34).

In 1996, Nakai reported from our laboratory an inhibitory effect of TPRO on gastric carcinogenesis induced by duodenogastric reflux (30). He made a rat model of duodenogastric reflux and administered a commercial diet containing $0.5 \%$ TPRO (TPRO group) and a commercial diet without additives (control group). At the 50th week after surgery the incidence of glandular stomach carcinoma of rats was $0 \%$ in the TPRO group, whereas it was $36 \%$ in the control group. The daily urinary excretion of NTPRO in rats was $2.0 \mu \mathrm{g}$ in the TPRO group and $<0.05 \mu \mathrm{g}$ in the control group. The results demonstrated that TPRO acts as a scavenger of nitrites and prevents carcinogenesis. This evidence led us to our present speculation that esophageal carcinogenesis may also be caused by endogenous nitrosation.

The sequential histological study disclosed 3 steps in the present carcinogenesis: firstly esophagitis; secondly CLE; and lastly AC. In the DGER control rats, the lower part of the esophagi exhibited the esophagitis featuring erosion, regenerative thickening and basal cell hyperplasia at the 25th week after surgery. The CLE and AC were first observed at the 25 th and 35th week, respectively. The incidence of CLE and AC sequentially increased after surgery and reached 89 and $44 \%$ at the 45 th week, respectively. This suggested the esophageal carcinogenesis as an inflammation-metaplasiaadenocarcinoma sequence.

It is not clearly understood which step of the experimental esophageal carcinogenesis can be prevented. Our laboratory has presented two studies on how to prevent this carcinogenesis. Nishijima et al made it clear that a switch procedure from the duodenoesophageal reflux model into the Roux-en-Y type model can stop the development of esophageal AC from CLE (35). Oyama et al reported that a COX-2 inhibitor, celecoxib, alleviates esophagitis to result in the inhibition of CLE and AC occurrence in rats (36).

Present sequential observation demonstrated erosion, regenerative thickening and basal cell hyperplasia in all of the rats at each periodic examination after surgery and did not show any difference in the incidence between the control and the TPRO groups. The occurrence of CLE was not different between the TPRO and the control groups. The incidence of AC was significantly depressed in the TPRO group at the 45th week after surgery as compared with the control group, though that of CLE was not different. This implied that TPRO prevented the progression from CLE to esophageal AC. Contrarily, Kumagai et al (31), have reported that TPRO reduced the occurrence of esophageal adenocarcinoma in rats which had undergone a side-to-side anastomosis between the jejunum and the esophagogastric junction 70 weeks prior to being sacrificed. In this rat model of duodenoesophageal reflux, the incidence of associated lesions such as esophageal ulcers and specialized columnar epithelium were lower in the TPRO group than in the control group, though there was no statistically significant difference. The occurrence of every pathological finding in the respective control groups was higher in our case than in the case of Kumagai, though the observation period for our case was 
shorter than in the case of Kumagai (31). This discrepancy can not be clearly explained, but we believe it may be attributable to different levels of intensity of DGER, said difference in levels perhaps resulting from the different methods of surgical intervention employed.

The question then arises whether TPRO may be applied to prevention of AC in humans. Goldstein et al described the pathological features of adenocarcinogenesis in rat esophagi as being similar to that in humans (11). We hypothesize that TPRO also may inhibit the formation of carcinogenic NOC in humans, and may also have effects on CLE (Barrett's esophagus) patients.

TPRO has been marketed in France since 1964 for the treatment of hepatic and biliary disorders (37) and has been administered to patients with advanced squamous cell carcinoma in the head and neck (38). However, due to suspected side-effects and toxicity of this drug (39), we have never been able to use it due to government restrictions. Though the clinical use of TPRO itself cannot be put into practice, suggestions for the further development of drugs suppressing the production of NOC are offered.

In immunohistochemical staining, iNOS and nitrotyrosine staining were positive in CLE in the DGER division. It is probable that nitric oxide is also related with carcinogenesis of esophageal $\mathrm{AC}$, because there are some reports that the expression level of iNOS is higher in esophageal AC than in non-neoplasmatic tissue in humans and rats $(24,25)$. Nitrites also promote tyrosine nitration through formation of nitryl chloride and nitrogen dioxide by myeloperoxidase (40). Since nitrotyrosine is a stable nitration product of tyrosine residue, it can be used as a marker for peroxynitrite and other nitrating species (41). This study showed that the stain of iNOS and nitrotyrosine were not suppressed by TPRO. Though reactive nitrogen substances could be associated with adenocarcinogenesis of the esophagus, more studies are necessary to investigate whether TPRO suppresses nitrating or not.

Miwa et al reported that bile was a more important component for the development of gastric carcinoma than pancreatoduodenal secretion (42). On the other hand, some groups of investigators concluded pancreatic exocrine secretion is the factor responsible for gastric (43) and esophageal (44) carcinogenesis. Pera et al reported that esophageal carcinomas were induced only when pancreatic secretion was present in the duodenal-content reflux together with low dose carcinogens (45), and that both pancreatobiliary and pancreatic secretion stimulated an expansion of the proliferative compartment of the esophageal squamous epithelium in rats (46). Nevertheless, we have found no reports that pancreatic juice is carcinogenic or mutagenic. Our results lead us to suppose that NOCs, such as $N$-nitrosoglycocholic acid and $N$-nitrosotaurocholic acid, may be related to the carcinogens of esophageal AC. Although no presence of NOCs including $N$-nitrosoglycocholic acid and $N$-nitrosotaurocholic acid could be confirmed in the duodenal juice of the rats with esophagojejunostomy (47), the unstable nature of $N$-nitroso compounds may account for the difficulty in detecting NOCs.

In conclusion, AC developed in rats with duodenogastroesophageal reflux and did not in those with gastroesophageal reflux. The oral administration of $0.5 \%$ TPRO inhibited the process of development from CLE to AC in this esophageal carcinogenesis. This implies that the carcinogenesis is related to endogenous duodenal nitrosation, and TPRO has been shown to exhibit a potent role in preventing reflux-induced esophageal adenocarcinogenesis in rats.

\section{References}

1. Devesa SS, Blot WJ and Fraumeni JF Jr: Changing patterns in the incidence of esophageal and gastric carcinoma in the United States. Cancer 83: 2049-2053, 1998.

2. Pera M, Cameron AJ, Trastek VF, Carpenter HA and Zinsmeister AR: Increasing incidence of adenocarcinoma of the esophagus and esophagogastric junction. Gastroenterology 104: 510-513, 1993.

3. Hongo M and Shoji T: Epidemiology of reflux disease and CLE in East Asia. J Gastroenterol 38 (Suppl 15): 25-30, 2003.

4. Cameron AJ, Otto BJ and Payne WS: The incidence of adenocarcinoma in columnar-lined (Barrett's) esophagus. New Engl J Med 313: 857-859, 1985.

5. Attwood SEA, DeMeester TR, Bremnar CG, Barlow AP and Hinder RA: Alkaline gastroesophageal reflux: implication in the development of complication in Barrett's columnar lower esophagus. Surgery 106: 764-770, 1989.

6. Championg G, Richter JE, Vaezi MF, Singh S and Alexander R: Duodenogastroesophageal reflux: relationship to $\mathrm{pH}$ and importance in Barrett's esophagus. Gastroenterology 107: 747-754, 1994.

7. Kauer WK, Peters JH, DeMeester TR, Ireland AP, Bremnar CG and Hagen JA: Mixed reflux of gastric and duodenal juices is more harmful to esophagus than gastric juice alone. The need for surgical therapy re-emphasized. Ann Surg 222: 525-531, 1995.

8. Melo LL, Kruel CDP, Kliemann LM, et al: Influence of surgical induced gastric and gastroduodenal content reflux on esophageal carcinogenesis - experimental model in Wistar female rats. Dis Esophagus 12: 106-115, 1999.

9. Miwa K, Sahara H, Segawa M, et al: Reflux of duodenal or gastro-duodenal contents induce esophageal carcinoma in rats. Int J Cancer 67: 269-274, 1996.

10. Miwa K, Segawa M, Takano Y, et al: Induction of esophageal and forestomach carcinomas in rats by reflux of duodenal contents. Br J Cancer 70: 185-189, 1994.

11. Goldstein SR, Yang G, Curtis SK, et al: Development of esophageal metaplasia and adenocarcinoma in a rat surgical model without the use of a carcinogen. Carcinogenesis 18: 2265-2270, 1997.

12. Fein M, Peters JH, Chandrasoma P, et al: Duodenoesophageal reflux induces esophageal adenocarcinoma without exogenous carcinogen. J Gastrointest Surg 2: 260-268, 1998.

13. Pera M, Brito M, Pera M, et al: Duodenal-content reflux esophagitis induces the development of glandular metaplasia and adenosquamous carcinoma in rats. Carcinogenesis 21: $1587-1591,2000$

14. Mirvish SS, Huang Q, Chen SC, et al: Metabolism of carcinogenic nitrosoamines in the rat and human esophagus and indication of esophageal adenocarcinoma in rats. Endoscopy 25 : 627-631, 1993.

15. Watt PC, Sloan JM, Donaldoson JD, Patterson CC and Kennedy TL: Relationship between histology and gastric juice $\mathrm{pH}$ and nitrite in the stomach after operation for duodenal ulcer. Gut 25: 246-252, 1984.

16. Correa P, Haenszel W, Cuello C, Tannenbaum S and Archer M: A model for gastric cancer epidemiology. Lancet 12: 58-60, 1975.

17. Mirvish SS: Formation of $N$-nitroso compounds: chemistry, kinetics and in vivo occurrence. Toxicol Appl Pharmacol 31: 325-351, 1975.

18. Calmels S, Bereziat JC, Ohshima $\mathrm{H}$ and Bartsch H: Bacterial formation of $N$-nitroso compounds from administered precursors in the rat stomach after omeprazole induced achlorhydria. Carcinogenesis 12: 435-439, 1991

19. Suzuki K and Mitsuoka T: $N$-nitrosoamine formation by intestinal bacteria. IARC Sci Publ 57: 275-281, 1984.

20. Mirvish SS: Role of $N$-nitroso compounds (NOC) and $N$-nitrosation in etiology of gastric, esophageal, nasopharyngeal and bladder cancer and contribution to cancer of known exposures to NOC. Cancer Lett 93: 17-48, 1995. 
21. Lee JS, Oh TY, Ahn BO, et al: Involvement of oxidative stress in experimentally induced reflux esophagitis and Barrett's esophagus: clue for the chemoprevention of esophageal carcinoma by antioxidants. Mutat Res 480-481: 189-200, 2001.

22. Wetscher GJ, Hinder RA, Bagchi D, et al: Reflux esophagitis in humans is mediated by oxygen-derived free radical. Am J Surg 170: 552-557, 1995.

23. Wetscher GJ, Perdikis G, Ketchmar DH, et al: Esophagitis in Sprague-Dawley rats is mediated by free radicals. Dig Dis Sci 40: 1297-1305, 1995.

24. Wilson KT, Fu S, Ramanujam KS and Meltzer SJ: Increased expression of inducible nitric oxide synthase and cyclooxygenase- 2 in Barrett's esophagus and associated adenocarcinomas. Cancer Res 58: 2929-2934, 1998.

25. Goldstein SR, Yang GY, Chen X, Curtis SK and Yang CS: Studies of iron deposits, inducible nitric oxide synthase and nitrotyrosine in a rat model for esophageal adenocarcinoma. Carcinogenesis 19: 1445-1449, 1998.

26. Ratner S and Clarke HT: The action of Formaldehyde upon cysteine. J Am Chem Soc 59: 200-206, 1937.

27. Tahira T, Tsuda M, Wakabayashi K, Nagao M and Sugimura T: Kinetics of nitrosation of thioproline, the precursor of a major nitroso compound in human urine, and its role as a nitrite scavenger. GANN 75: 889-894, 1984

28. Weber HU, Fleming JF and Miquel J: Thiazolidine-4-carboxylic acid, a physiologic sulfhydryl antioxidant with potential value in geriatric medicine. Arch Gerontol Geriatr 1: 299-310, 1982.

29. Tahira T, Ohgaki H, Wakabayashi K, Nagao M and Sugimura T: The inhibitory effect of thioproline on carcinogenesis induced by $N$-benzylmethylamine and nitrite. Fd Chem Toxic 26: 511-516, 1988.

30. Nakai M: Inhibitory effect of Thioproline on gastric carcinogenesis in rats with surgically induced duodenogastric reflux. Journal of the Juzen Medical Society 105: 648-657, 1996 (In Japanese).

31. Kumagai H, Mukaisho K, Sugihara H, Miwa K, Yamamoto G and Hattori T: Thioproline inhibits development of esophageal adenocarcinoma induced by gastroduodenal reflux in rats. Carcinogenesis 25: 723-727, 2004.

32. Sato T, Miwa K, Sahara H, Segawa M and Hattori T: The sequential model of Barrett's esophagus and adenocarcinoma induced by duodeno-esophageal reflux without exogenous carcinogens. Anticancer Res 26: 565-570, 2005.
33. Mirvish SS: The etiology of gastric cancer. J Natl Cancer Inst 71: 629-647, 1983.

34. Busby WF, Shuker DEG, Charnley G, Newberne PM, Tannenbaum SR and Wogen GN: Carcinogenicity in rats of the nitrosated bile acid and conjugates $N$-nitrosoglycocholic acid and $N$-nitrosotaurocholic acid. Cancer Res 45: 1367-1371, 1985.

35. Nishijima K, Miwa K, Miyashita T, et al: Impact of biliary diversion procedure on carcinogenesis in Barrett's esophagus surgically induced by duodenoesophageal reflux in rats. Ann Surg 240: 57-67, 2004.

36. Oyama K, Fujimura T, Ninomiya I, et al: A COX-2 inhibitor prevents the esophageal inflammation-metaplasia-adenocarcinoma sequence in rats. Carcinogenesis 26: 565-570, 2005.

37. Garnier R, Efthymiou ML and Fournier E: Acute thioproline poisoning. J Toxicol Clin Toxcol 19: 289-295, 1982.

38. Brugarolas A and Gosalvez M: Treatment of cancer by an inducer of reverse transformation. Lancet 1: 68-70, 1980.

39. Nasca S, Galichet V, Jardillier JC, Garbe E and Coninx P: Thioproline toxicity. Lancet 1: 778, 1981 .

40. Eiserich JP, Hristova M, Cross CE, et al: Formation of nitric oxide-derived inflammatory oxidants by myeloperoxidase in neutrophils. Nature 391: 393-397, 1998.

41. Crow JP and Ischiropoulos S: Detection and quantization of nitrotyrosine residue in proteins: in vivo marker of peroxynitrite. Methods Enzymol 269: 185-194, 1996.

42. Miwa K, Fujimura $\mathrm{T}$, Hasegawa $\mathrm{H}$, et al: Is bile or are pancreaticoduodenal secretions related to gastric carcinogenesis in rats with reflux through the pylorus? J Cancer Res Clin Oncol 118: 570-574, 1992.

43. Mason RC: Duodenogastric reflux in rat gastric carcinoma. Br J Surg 73: 801-803, 1986.

44. Yamashita Y, Homma K, Kako N, et al: Effect of duodenal components of the refluxate on development of esophageal neoplasia in rats. J Gastrointest Surg 2: 350-355, 1998.

45. Pera M, Trastek VF, Carpenter HA, et al: Influence of pancreatic and biliary reflux on the development of esophageal carcinoma. Ann Thorac Surg 55: 1386-1393, 1993.

46. Pera M, Grande L, Gelabert M, et al: Epithelial cell hyperproliferation after biliopancreatic reflux into the esophagus of rats. Ann Thorac Surg 65: 779-786, 1998.

47. Fein M, Fuchs KH, Stopper H, Diem S and Herderich M: Duodenogastric reflux and foregut carcinogenesis: analysis of duodenal juice in a rodent model of cancer. Carcinogenesis 21 : 2079-2084, 2000 